\title{
Otimização e validação de metodologia para determinação de hormônios sexuais em matrizes aquosas por SPE e GC/MS: determinação de hormônios sexuais em matrizes aquosas
}

Os hormônios sexuais têm sido considerados contaminantes emergentes do ecossistema aquático e podem desencadear sérios problemas na base da cadeia alimentar. O objetivo deste estudo foi otimizar uma metodologia analítica, envolvendo extração em fase sólida (SPE) com C18 de Estriol, Estradiol e Testosterona em matrizes aquosas e sua determinação por cromatografia gasosa acoplada a espectrometria de massa (GC/MS). O estudo foi desenvolvido utilizando amostras aquosas fortificadas, sendo avaliados: volume de amostra, $\mathrm{pH}$, volume de eluição com diclorometano no processo de SPE. Para avaliar a metodologia otimizada, foram analisadas amostras de esgoto sanitário fortificado e não fortificado. 0 método mostrou precisão (RSD 8,6 e 12,4\%), recuperação (121,2 e 120,6\%), LDs $(0,022$ e $0,015 \mu \mathrm{g}$ L-1) e LQs (0,073 e 0,053 $\mu \mathrm{g} \mathrm{L-1)}$, aceitos para as faixas de $\mu \mathrm{g} \mathrm{L-1}$ (para estradiol e testosterona), o estriol apresentou recuperações de $48,2 \%$ e RSD de $20,7 \%$. A aplicação da metodologia em amostras reais permitiu detectar a presença de testosterona e estradiol nas amostras de efluentes de esgoto doméstico.

Palavras-chave: Contaminantes emergentes; Disruptores endócrinos; Extração em fase sólida.

\section{Optimization and validation of methodology for determining sex hormones in aqueous matrices by SPE and GC/MS: determination of sex hormones in aqueous matrices}

\begin{abstract}
Sex hormones have been considered contaminants emerging from the aquatic ecosystem, and can trigger serious problems at the base of the food chain. The objective of this study was to optimize analytical methodology, involving solid phase extraction (SPE) with C18 of Estriol, Estradiol and Testosterone in aqueous matrices and its determination by gas chromatography coupled with mass spectrometry (GC/MS). The study was developed using aqueous fortified samples, being evaluated: volume de amostras, $\mathrm{pH}$, volume de eluição com diclorometano no processo de SPE. To evaluate the optimized methodology, samples of fortified and unfortified sanitary sewage were analyzed. The method showed precision (RSD 8.6 and $12.4 \%$ ), recovery (121.2 and $120.6 \%)$, LDs (0.022 and $0.015 \mu \mathrm{g}$ L-1), and LQs $(0.073$ and $0.053 \mu \mathrm{g} \mathrm{L}-1)$ accepted for the $\mu \mathrm{g} \mathrm{L-1}$ ranges (for estradiol and testosterone), estriol showed recoveries of $48.2 \%$ and RSD of $20.7 \%$. The application of the methodology to real samples allowed to detect the presence of testosterone and estradiol in the sewage effluent samples.
\end{abstract}

Keywords: Emerging contaminants; Endocrine disruptors; Solid phase extraction

Topic: Desenvolvimento, Sustentabilidade e Meio Ambiente

Reviewed anonymously in the process of blind peer.
Received: $\mathbf{1 0 / 0 8 / 2 0 2 0}$

Approved: 21/09/2020
Flávia dos Santos Ferreira

Instituto Federal Sul-Rio-Grandense, Brasil

http://lattes.cnpq.br/0413779315833374

f.ferreira1892@gmail.com

Pedro José Sanches Filho (iD

Instituto Federal Sul-Rio-Grandense, Brasil

http://lattes.cnpq.br/9785390634457316

http://orcid.org/0000-0002-9852-8940

pjsans@ibest.com.br
Referencing this:

FERREIRA, F. S.; SANCHES FILHO, P. J.. Otimização e validação de metodologia para determinação de hormônios sexuais em matrizes aquosas por SPE e GC/MS: determinação de hormônios sexuais em matrizes aquosas. Revista Ibero Americana de Ciências Ambientais, v.11, n.5, p.500-509, 2020. DOI: http://doi.org/10.6008/CBPC2179$\underline{6858.2020 .005 .0045}$ 
Otimização e validação de metodologia para determinação de hormônios sexuais em matrizes aquosas por SPE e GC/MS: determinação de

\section{INTRODUÇÃO}

Os hormônios são mensageiros químicos que respondem pela comunicação entre diferentes tipos de células. Dentre os hormônios sexuais, os estrógenos vêm recebendo bastante destaque em estudos, por serem extremamente ativos biologicamente e por estarem relacionados à etiologia de vários tipos de câncer (VIALI, 2014). A relevância dos hormônios estrogênicos na perturbação endócrina se deve: à alta afinidade dos estrógenos aos receptores presentes nos organismos de outras espécies permitindo a ação, mesmo em baixíssimas concentrações ( $\mathrm{ng} \mathrm{L}^{-1}$ ); à ampla excreção destes hormônios por seres humanos e por outros animais nas fezes e urina; e ao posterior despejo dos esgotos, tratados ou não, nos ecossistemas aquáticos (TEIXEIRA et al., 2018).

A testosterona é o principal hormônio sexual masculino e um esteroide anabolizante. Em humanos e animais do sexo masculino, a testosterona desempenha um papel fundamental no desenvolvimento de tecidos reprodutores masculinos, como testículos e próstata, bem como a promoção de características sexuais secundárias, como o aumento da massa muscular, aumento e maturação dos ossos e o crescimento do cabelo corporal. Além disso, a testosterona está envolvida na saúde, no bem-estar e na prevenção da osteoporose. Níveis insuficientes de testosterona nos homens podem levar a anormalidades, incluindo fragilidade e perda óssea (TUCK et al., 2009).

O hormônio estradiol é um mediador de suma importância para o crescimento, diferenciação e função de diversos tecidos alvos, incluindo os sistemas reprodutivos masculinos e femininos, a glândula mamária e os sistemas cardiovascular e esquelético (COSTA, 2018). Na pré-menopausa, em mulheres saudáveis, mais de $90 \%$ do estradiol é sintetizado pelos ovários, onde a androstenediona, seu percursor, é convertido em estrona e posteriormente metabolizado em estradiol. Esta conversão é mediada por aromatases da família do citocromo $\mathrm{P} 450$, podendo este processo ocorrer nos tecidos extragonadais como o cérebro, músculo, osso, tecido adiposo e na mama (MORSELLI et al., 2017).

O Estriol é um estrógeno natural que está presente na circulação sanguínea das mulheres e aumenta suas concentrações plasmáticas durante a gravidez. Trata-se de um metabólito oriundo da oxidação do Estradiol. No que diz respeito à ocorrência ambiental, tem sido detectado em diversos países (DALLEGRAVE, 2013). Com a crescente preocupação ambiental científica, quanto à presença dessas substâncias no meio ambiente e seus efeitos cada vez maiores, muitos pesquisadores têm otimizado metodologias para o desenvolvimento de análise em matrizes ambientais. Busca-se desta forma metodologias que permitam o controle dos níveis destes contaminantes, para que sirvam de ferramentas em estudo de degradação e monitoramento destes hormônios em efluentes e ambientes hídricos que possam vir a receber estes contaminantes, como rios e lagos (SOUZA et al., 2015).

Basicamente, a análise de hormônios sexuais compreende as etapas de extração e pré-concentração do analito, eliminação de interferentes, separação, detecção e sua quantificação. As técnicas analíticas mais utilizadas para quantificação de hormônios sexuais em amostras ambientais estão fundamentadas em técnica cromatográfica (SOUZA et al., 2015). Dentre elas destaca-se a cromatografia gasosa com detector de 
Otimização e validação de metodologia para determinação de hormônios sexuais em matrizes aquosas por SPE e GC/MS: determinação de

massas (do inglês gas chromatography coupled to mass spectrometry GC/MS), cromatografia gasosa com espectrômetro de massa em série (GC/MS²) (HE et al., 2019), cromatografia líquida com detector por arranjo de diodos (LC-DAD) (QUEIROZ et al., 2013) e cromatografia líquida com espectrometria de massas (LC-MS) (GOEURY et al., 2019).

Estas técnicas necessitam de etapas de extração, pré concentração e clean up em função dos níveis de concentração dos analitos, presença de interferentes e efeito de matrizes nas amostras, desta forma, o objetivo deste estudo foi otimizar metodologia analítica, envolvendo extração em fase sólida (SPE) com C18 de Estriol, Estradiol e Testosterona, em matrizes aquosas e a sua determinação por cromatografia gasosa acoplada a espectrometria de massas (GC/MS) utilizando derivatização com MSTFA (N-metil-N-trimetilsililtrifluoroacetamida).

\section{MATERIAIS E MÉTODOS}

\section{Material, Soluções e Reagentes}

Foram utilizados para o preparo das soluções de trabalho: Estradiol Base, marca Infinity Pharma, grau de pureza 97\%; Estriol Base, marca Infinity Pharma, grau de pureza 97\%, Testosterona Base, marca Infinity Pharma, grau de pureza 97\%; Solventes: Acetonitrila, grau de pureza 99,98\% (Merck), Metanol, grau de pureza 99,99\% (Merck), Diclorometano, grau de pureza 99,99\% (Merck), todos destilados; água ultrapura obtida através de sistema de purificação MiliQ (Milipore Co). Cartuchos para SPE C18 (Chromabond ${ }^{\circledR}$ EC 6,0 $\mathrm{mL} / 1000 \mathrm{mg}$ ) foram utilizados para otimização da extração em fase sólida, adquiridos da Sulpelco (Bellefonte, PA, USA). Para a derivatização foi utilizado N-metil-N-trimetilsilil-trifluoroacetamida (MSTFA, Sigma Aldrich), grau de pureza 98,5\%, em ampolas de $1,0 \mathrm{~mL}$.

As soluções estoques individuais de cada analito (Estradiol, Estriol, Testosterona), foram preparadas pesando- se $200 \mathrm{mg}$ de cada analito e avolumando-as, o estradiol e a testosterona com acetonitrila e o estriol com metanol a 10,0 mL obtendo-se soluções estoques a $20 \mathrm{~g} \mathrm{~L}^{-1}$. As soluções de trabalho foram preparadas a partir de diluições, desta solução, em acetonitrila para que o metanol não reagisse com o agente derivatizante, afetando a etapa de derivatização. As soluções foram estocadas em um freezer $a-4^{\circ} \mathrm{C}$.

\section{Análise cromatográfica - GC/MS}

Para determinação dos compostos foi utilizado um cromatógrafo a gás acoplado a um espectrômetro de massas da Shimadzu, modelo GC/MS - QP2010 Ultra, equipado com uma coluna OV-5MS (30,0 m x 0,25 $\mathrm{mm} \times 0,25 \mu \mathrm{m}$ ), com uma temperatura de injeção de $250^{\circ} \mathrm{C}$, volume de injeção de $1 \mu \mathrm{L}$ pelo modo SPLITLESS, com gás de arraste Hélio com fluxo de $1,0 \mathrm{~mL} \mathrm{~min}^{-1}$, com $280{ }^{\circ} \mathrm{C}$ de temperatura na interface e $200{ }^{\circ} \mathrm{C}$ na fonte de íons. Os espectros de massas foram obtidos através eletro-ionização a $70 \mathrm{eV}$. A programação do forno foi variada conforme a Tabela 1, sendo o espectrômetro de massas operado em modo SCAN e SIM. 


\section{Derivatização}

Todo o extrato a ser derivatizado foi transferido para frascos de $1,0 \mathrm{~mL}$, onde foram adicionados 0,5 $\mathrm{mL}$ de diclorometano, e $40 \mu \mathrm{L}$ de derivatizante (MSTFA), passando por um banho de areia a $80^{\circ} \mathrm{C}$ por 30 minutos, esfriado e avolumado para 1,0 mL para posterior análise por GC/MS segundo Orata (2012). Os hormônios passaram a ser referidos neste estudo como Hormônio TMS.

\section{Extração em fase sólida - SPE}

Foi empregada uma solução aquosa de $50 \mu \mathrm{g} \mathrm{L}^{-1}$ a partir da diluição da solução estoque de $2000 \mathrm{mg}$ $\mathrm{L}^{-1} \mathrm{em}$ acetonitrila da mistura dos três analitos. Para o acondicionamento dos cartuchos $\mathrm{C} 18$, utilizou-se 2,0 $\mathrm{mL}$ de metanol seguido de 1,0 $\mathrm{mL}$ de água ultrapura, e como eluente utilizou-se diclorometano. Foram avaliados volume de amostra (100 e $200 \mathrm{~mL}$ ), pH de 3,0 e 7,0 da amostra (o ajuste de pH foi feito com uma solução de ácido clorídrico $0,1 \mathrm{~mol} \mathrm{~L}^{-1}$ ) e volume de eluição 5,0 e 10,0 mL com diclorometano. Para a otimização, foi verificado a precisão do método, através do desvio padrão relativo em porcentagem (RSD \%), calculado a partir dos resultados de repetições (triplicatas) do processo de SPE e recuperação considerando $50 \mu \mathrm{g} \mathrm{L} \mathrm{L}^{-1}$ como $100 \%$.

\section{Otimização do método cromatográfico}

Para a otimização do método cromatográfico foram preparadas soluções a $1000 \mathrm{mg} \mathrm{L}^{-1} \mathrm{em}$ acetonitrila de cada analito sendo derivatizados separadamente, cada solução estoque foi cromatografada isoladamente em modo SCAN, para determinação da ordem de saída através dos tempos de retenção, e identificação dos principais íons no espectro de massas. As condições cromatográficas foram adaptadas a partir de Dallegrave (2013). Posteriormente buscando melhora na resolução dos picos cromatográficos, foram variadas diferentes condições de programações de temperatura do forno, utilizando uma mistura dos analitos a $1000 \mathrm{mg} \mathrm{L}^{-1} \mathrm{em}$ diclorometano. As condições de análise do GC/MS estão apresentadas na Tabela 1.

A linearidade foi avaliada através da construção de curva de calibração numa faixa de 10 a $200 \mu \mathrm{gL}^{-1}$ dos padrões. Os estudos de precisão foram determinados através do cálculo do desvio padrão relativo das áreas obtidas a partir da repetição de 5 vezes do padrão de $100 \mu \mathrm{g} \mathrm{L}^{-1}$. Os limites de deteç̧ão (LD) e quantificação (LQ) foram calculados com base na análise do sinal de brancos considerando 3 vezes desvio padrão para LD e 10 vezes o desvio padrão para LQ e dividido pelo coeficiente angular das curvas analíticas respectivamente (IUPAC, 1997).

Tabela 1: Programação de temperatura para analises cromatográfica dos hormônios.

\begin{tabular}{|l|l|l|l|}
\hline Condições & $1^{\circ}$ programação & $2^{\circ}$ programação & $3^{\circ}$ programação \\
\hline Rampa & $150^{\circ} \mathrm{C}(2 \mathrm{~min})-10^{\circ} \mathrm{C} / \mathrm{min}-300^{\circ} \mathrm{C}$ & $200^{\circ} \mathrm{C}(0 \mathrm{~min})-5^{\circ} / \mathrm{min}-300^{\circ} \mathrm{C}$ & $150^{\circ} \mathrm{C}(0 \mathrm{~min})-5^{\circ} \mathrm{C} / \mathrm{min}-300^{\circ} \mathrm{C}$ \\
aquecimento & $(15 \mathrm{~min})$ & $(15 \mathrm{~min})$ & $(15 \mathrm{~min})$ \\
\hline Modo de leitura & SCAN & SCAN & SCAN \\
\hline
\end{tabular}

SCAN - Varredura total para m/z entre 35 a 500; SIM Select lon Monitoring 


\section{Aplicação da metodologia em amostra real}

Após a otimização de todas as etapas e para avaliar os efeitos de matriz, a metodologia foi aplicada a 3 amostras de efluentes de esgoto: Efluente esgoto bruto (EEB); Efluente de esgoto tratado (EET); Efluente de esgoto tratado fortificado (EETF).

As amostras de efluente de esgoto foram obtidas, na lagoa de estabilização que possui uma área de quatro hectares e sua capacidade é de $7.500 \mathrm{~m}^{3}$ por dia. Esta lagoa tem o objetivo de minimizar a carga poluidora dos efluentes de esgotos das residências e indústrias. Onde os resíduos são tratados antes de serem lançados no Arroio Santa Bárbara e após para o Canal São Gonçalo. As amostras de efluente foram coletadas $(500 \mathrm{~mL}$ ) na entrada e na saída das lagoas de estabilização. Amostragem foi realizada em abril de 2019 , com o auxílio de frascos de vidro, os quais foram acondicionados sob-refrigeração a $-4^{\circ} \mathrm{C}$ e transportados até o laboratório. As amostras antes das análises foram filtradas com papel filtro, para que se retirassem os resíduos sólidos suspensos. As amostras EETF foram obtidas através da fortificação da amostra da saída da lagoa de estabilização com $50 \mathrm{\mu g} \mathrm{L}^{-1}$ partir da solução estoque de $2000 \mathrm{mg} \mathrm{L}^{-1} \mathrm{em}$ acetonitrila da mistura dos três analitos. Os ensaios foram feitos em triplicatas acompanhados de análises de brancos.

\section{RESULTADOS E DISCUSSÃO}

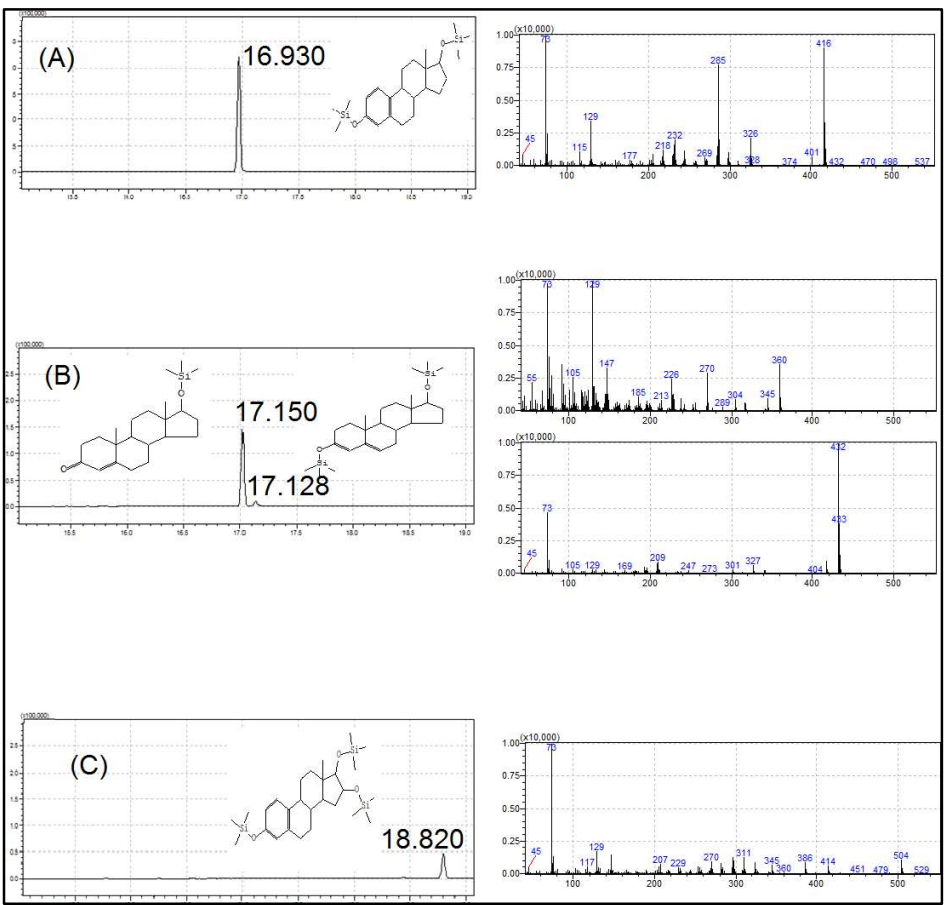

Figura 1: Cromatogramas modo e espectro de massas em modo SCAN: (A) Estradiol TMS, (B), Testosterona TMS e (C) Estriol TMS.

Com base no estudo cromatográfico de cada analito, isolado e em modo SCAN, foi possível determinar ordem de saída de cada Hormônio TMS, a Figura 1 apresenta o cromatograma para cada composto com os tempos de retenção e seus respectivos espectros de massa. Selecionaram-se os íons com razão massa/carga (m/z) 416 e 285, 432 e 360 e 311 e 504 para estradiol, testosterona e estriol, sendo estes utilizados para o modo SIM. Foi possível observar que o padrão de testosterona após a derivatização 
constituía-se de uma mistura de Androsterona ( $\operatorname{Tr} 17,028 \mathrm{~min}$ ) e Testosterona $(\operatorname{Tr} 17,128)$ sendo neste trabalho, tratadas através da soma das áreas e expressas como testosterona.

Inicialmente foram utilizadas as condições descritas por Dallegrave (2013), descrita programa 1 temperatura expressa na tabela 1 por CG/MS. O cromatograma obtido está apresentado na Figura 2A, o composto 1 estradiol TMS apresentou um tr de 16,976 minutos seguido da testosterona e androsterona (composto $2 \mathrm{a}$ em 17,023 min e composto $2 \mathrm{~b}$ em 17,143 $\mathrm{min}$ ) e o composto 3 estriol com tr de 18,806 min. (Figura 2)

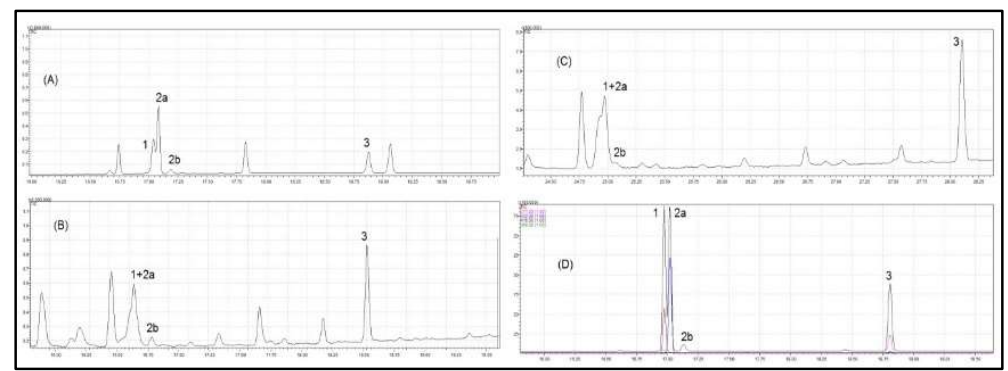

Figura 2: Cromatogramas para mistura de padrão derivatizado a $1000 \mathrm{mg} \mathrm{L}^{-1}$, durante a otimização do método cromatográfico.

Tentando reduzir os tempos de retenção, modificou-se a programação do forno, a elevação da temperatura $\left(200^{\circ} \mathrm{C}\right)$ promoveu a redução do tempo de retenção, mas, houve co-eluição e perda na resolução do estradiol e testosterona (Figura $2 \mathrm{~B}$ ), mesmo com rampa de temperatura mais suave (Figura $2 \mathrm{C}$ ).

Para o aumento da sensibilidade a detecção no espectrômetro de massas foi alterada do modo SCAN para modo SIM, empregando os íons $\mathrm{m} / \mathrm{z}$, previamente selecionados. Podemos verificar na Figura 2D os picos relativos aos íons m/z 432 do estradiol TMS, 416 da testosterona TMS, encontrando-se praticamente resolvidos, não interferindo nas determinações quantitativas, uma vez que estes são específicos de cada analito, um não está presente no espectro do outro. Também se observa o clean up eletrônico com o desaparecimento de alguns contaminantes. Estas condições reunidas foram utilizadas para as demais etapas do estudo. A tabela 2 apresenta as figuras de mérito para o método cromatográfico. Os dados demonstram que o método se comporta de forma linear com r2 de 0,995 para estradiol, 0,991 para testosterona e 0,998 para estriol.

Tabela 2: Figuras de mérito para o método cromatográfico otimizado.

\begin{tabular}{|l|l|l|l|l|l|}
\hline Hormônios TMS & Equação da reta & $\mathbf{R}^{\mathbf{2}}$ & $\mathbf{L D}\left(\boldsymbol{\mu \mathbf { g ~ L } ^ { - 1 } )}\right.$ & $\mathbf{L Q}\left(\boldsymbol{\mu \mathbf { g ~ L } ^ { - 1 } )}\right.$ & $\mathbf{R S D}(\%)$ \\
\hline Estradiol & $\mathrm{y}=40,6-15,1$ & 0,995 & 2,2 & 7,3 & 7,5 \\
\hline Testosterona & $\mathrm{y}=50,9-31,1$ & 0,991 & 1,5 & 5,1 & 5,4 \\
\hline Estriol & $\mathrm{y}=30,8+82,7$ & 0,998 & 1,8 & 5,9 & 5,1 \\
\hline
\end{tabular}

$r^{2}=$ coeficiente de correlação linear; $L D=$ limite de deteç̧ão; $L Q=$ limite de quantificação; RSD\%= desvio padrão relativo em \% (coeficiente de variação).

O limite de detecção (LD) e o limite de quantificação (LQ) encontrado foram: 2,2 e 7,3 $\mu \mathrm{g} \mathrm{L}^{-1}$ para o estradiol, 1,5 e 5,1 $\mu \mathrm{g} \mathrm{L}^{-1}$ para a testosterona e 1,8 e 5,9 $\mathrm{g} \mathrm{L} \mathrm{L}^{-1}$ para estriol, respectivamente. Estes valores são inferiores aos descritos por Araújo (2006), que encontrou LD 16,4 a $80 \mu \mathrm{g} \mathrm{L}^{-1}$ e LQ 49 a $250 \mu \mathrm{g} \mathrm{L}^{-1}$ para uma gama de hormônios estrogênios, utilizando cromatografia liquida e deteç̧ão ultravioleta. Os RSD \% indicaram que o método foi preciso, estando em uma faixa de 5,1\% a 7,5\%, segundo Wood (1999) valores 
Otimização e validação de metodologia para determinação de hormônios sexuais em matrizes aquosas por SPE e GC/MS: determinação de

menores que $20 \%$ são aceitos para esta faixa de concentração (10 a $200 \mu \mathrm{g} \mathrm{L^{-1 }}$ ), além disso, estes valores estão em acordo com Campos (2018) que encontrou valores 6,3\% a 7,6\% seguindo o critério do INMETRO (2016) que também estabelece valores aceitáveis menores 20\% para análise de substâncias em níveis traço por GC/MS.

\section{Otimização do SPE}

O volume de 100,0 mL garantiu recuperações 121,2\% e 120,6\% para o estradiol e testosterona, com um CV inferior $20 \%$ o que representa uma precisão aceitável. Para amostra com concentrações na ordem de $\mu \mathrm{L} \mathrm{L}^{-1}$ recuperações na faixa de 50 a $120 \%$ e CV de até $20 \%$ são adequadas segundo Brito et al. (2003) que apresenta estes valores em seu trabalho "Validação de métodos analíticos" e estão em acordo com as descritas por Filippe (2018) que encontrou 20 a 120\%, porém, para o estriol o método apresentou uma recuperação de $48,2 \%$ o que pode ser justificado por este composto apresentar 3 hidroxilas o que favorece sua interação com a matriz aquosa. Recuperações entre 40 e 101\% foram descritas por Silva et al. (2011), para determinação de CE utilizando a técnica de SPE para água de rio e efluentes domésticos.

Aumentado o volume para 200,0 mL observa-se uma queda das recuperações com valores inferiores a 40\%, acompanhados do aumentou dos valores de RSD\% (Tabela 3) indicando uma perda na repetibilidade do processo. Neste volume já ocorre à lixiviação dos compostos retidos na fase extratora (volume de Breakthrough). Novamente o estriol apresentou o menor valor de recuperação em função da sua polaridade sendo assim, o volume de amostra a ser percolado no processo de SPE com C18 foi definido em 100,0 mL.

Tabela 3: Recuperações do Método SPE com C18 em função do Volume e estudo de precisão

\begin{tabular}{|l|l|l|l|l|l|l|}
\hline Hormônios & $\mathbf{1 0 0}(\mathbf{m L})$ & $\mathbf{R S D}(\%)$ & $\mathbf{2 0 0}(\mathbf{m L})$ & $\mathbf{R S D}(\%)$ & $\mathbf{L} \mathbf{D}^{*}\left(\boldsymbol{\mu \mathbf { g ~ L } ^ { - 1 } )}\right.$ & $\mathbf{L Q}^{*}\left(\boldsymbol{\mu \mathbf { g ~ L } ^ { - 1 }}\right)$ \\
\hline Estradiol & 121,2 & 8,6 & 35,1 & 24,9 & 0,022 & 0,073 \\
\hline Testosterona & 120,6 & 12,4 & 40,3 & 23,5 & 0,015 & 0,051 \\
\hline Estriol & 48,2 & 20,7 & 2,3 & 35,9 & 0,018 & 0,059 \\
\hline
\end{tabular}

LD = limite de deteç̧ãoo; $L Q$ = limite de quantificação; RSD\%= desvio padrão relativo em \%, (coeficiente de variação). * calculado para $100,0 \mathrm{ml}$ de amostra.

Quanto ao estudo dos volumes de eluição dos analitos adsorvidos, estes foram detectados apenas nos primeiros $5 \mathrm{~mL}$ de DCM, ficando definido, portanto, o volume de $5,0 \mathrm{~mL}$ deste solvente. Quando comparados os resultados de recuperação utilizando $100,0 \mathrm{~mL}$ da solução aquosa com o pH 3 e pH 7, não se observou alteração. Portanto optou-se por utilizar pH 3 que restringe a ionização dos hormônios, como descrito na bibliografia (DALLEGRAVE, 2013).

Para o método SPE com C18 otimizado, os LD's variam entre 0,015 a 0,022 e para os LQ's variam entre 0,051 a 0,073, estando na mesma faixa como descrita em outros estudos. Baronti et al. (2000), determinou estriol em níveis de 24 - $188 \mathrm{ng} \mathrm{L}^{-1}$ em esgoto doméstico na Itália, o estradiol foi encontrado de 0,5 - 17,0 $\mathrm{ng} \mathrm{L}^{-1} \mathrm{em}$ esgoto doméstico na Itália e Holanda (JONHSON et al., 2000) e 0,021 $\mathrm{\mu gL}^{-1} \mathrm{em}$ esgoto doméstico no Brasil (TERNES et al., 1999) e Kolpin et al. (2002) encontrou 0,116 $\mu \mathrm{g} \mathrm{L}^{-1}$ de testosterona em água natural nos EUA. 
Otimização e validação de metodologia para determinação de hormônios sexuais em matrizes aquosas por SPE e GC/MS: determinação de

\section{Aplicação da metodologia em amostra real}

A Figura 3A compara os cromatogramas resultantes das análises das amostras EETF e EET em modo SCAN, pode-se verificar a presença dos picos referentes aos analitos no cromatograma EETF que não foram detectados no EET, além de uma série de outros compostos co-extraídos. Estes compostos co-extraídos podem explicar a influência da matriz, o que foi comprovado através da redução nas recuperações dos hormônios descritas na tabela 3 (121, 2\%; 120,6\% e 48,2\%) para 71,4\%; 40,3\%; e 27,7\% para estradiol, testosterona e estriol, respectivamente (tabela 4). Recuperações de $71,4 \%$ ainda são consideras aceitáveis para estradiol (BRITO, 2003) (tabela 4), estando em acordo com recuperações encontradas por Jauković, et al., (2017), onde obteve recuperações entre 70\% - 114\% de hormônios esteroides em águas residuais.

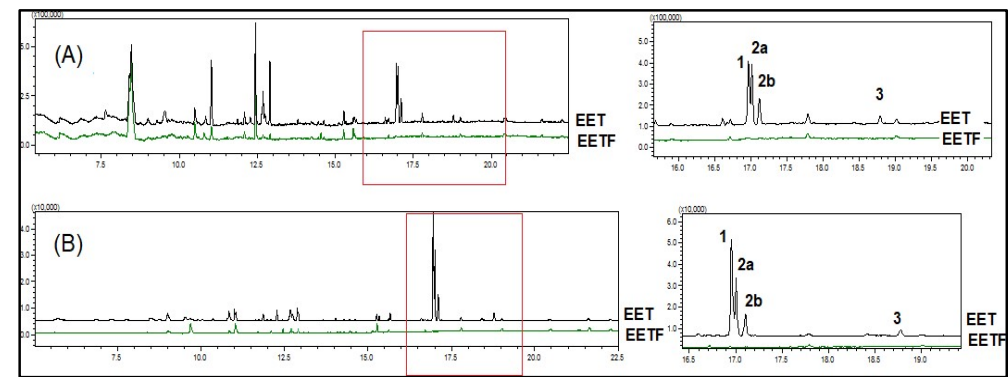

Figura 3: Cromatogramas dos extratos de efluente esgoto tratado (EET) e efluente esgoto tratado fortificado EETF. (A) modo SCAN; (B) modo SIM.

O estudo nas amostras reais também apresentou precisões adequadas com RSD\% abaixo de $20 \%$. A redução da recuperação pode ser justificada pela concorrência nos sítios ativos do SPE com outros compostos presentes na matriz. O grupo octadecil ligados a sílica (C18) interagem com compostos orgânicos hidrofóbicos pela ação das forças de Van der Waals (CHRISTIAN, 1994) estes resultados indicam que estudos futuros de otimização aumentando a massa de adsorvente podem vir a melhorar estas recuperações.

A Figura 3B compara os cromatogramas resultantes das análises das amostras EETF e EET em modo SIM. Esta imagem evidência o clean up eletrônico produzido neste modo de análise. Comparando a figura 3B com a 3A observa-se uma redução nos picos da matriz, tanto na quantidade quanto na intensidade. $O$ aumento da área dos picos dos analitos em relação aos picos da matriz comprova o aumento da sensibilidade promovida pela análise cromatográfica em modo SIM. A Tabela 4 apresenta os resultados para os níveis de hormônios em amostras reais (EETF, EET, EEB) bem como o desvio padrão relativo (\%). Foi possível quantificar os analitos apenas nas amostras EETF.

Tabela 4: Níveis de Hormônios em amostras reais.

\begin{tabular}{|l|l|l|l|l|l|}
\hline Hormônios & EETF $\left(\mu \mathbf{g ~ L}^{-1}\right)$ & RSD $(\%)$ & REC & EEB & EET \\
\hline Estradiol & 35,7 & 7,5 & 71,4 & D & D \\
\hline Testosterona & 20,2 & 20,0 & 40,3 & D & D \\
\hline Estriol & 13,8 & 18,0 & 27,7 & ND & ND \\
\hline
\end{tabular}

EETF: efluente esgoto trato fortificado; REC: recuperação; RSD\%= desvio padrão relativo em \% (coeficiente de variação). ; EEB: efluente esgoto bruto; EET: efluente esgoto tratado D: detectado; ND: não detectado.

Ao aplicar a metodologia otimizada, SPE com C18 e GC/MS em modo SIM, às amostras EEB e EET foi possível detectar a presença de estradiol e testosterona em ambas, numa faixa de $0,02 \mu \mathrm{g} \mathrm{L}^{-1}$ a $0,07 \mu \mathrm{g} \mathrm{L}^{-1}$ 
para o estradiol e $0,02 \mu \mathrm{g} \mathrm{L}^{-1}$ a 0,05 $\mu \mathrm{g} \mathrm{L}^{-1}$ para testosterona (os níveis destes analito situaram-se entre LD e o LQ descritos na tabela 3. Estes resultados indicam ineficiência da lagoa de estabilização na redução destes analitos uma vez que foram detectados na mesma faixa em amostras obtidas na entrada (EEB) e na saída da lagoa (EET).

Estes compostos foram descritos por outros autores em amostras de efluentes de esgoto, como Pessoa et al. (2014) que determinou concentrações de hormônios sexuais em regiões de seca, no Ceará na faixa de 0,776 $\mu \mathrm{g} \mathrm{L}^{-1}$ a 3,180 $\mu \mathrm{g} \mathrm{L}^{-1}$, já as concentrações encontradas por Ferreira (2013) em esgoto do Rio de Janeiro estiveram na faixa de $\mathrm{ng} \mathrm{L}^{-1} \mathrm{em}$ acordo com os resultados apresentados neste estudo.

\section{CONCLUSÕES}

A metodologia otimizada neste estudo, combinando extração em fase sólida (SPE) com C18, derivatização com MSTFA e quantificação no modo SIM por GC/MS apresentou potencial para determinação tanto da testosterona como estradiol em efluentes de esgoto sanitário podendo ser aplicada a outros analitos com estrutura química similar. O método apresentou precisão, recuperação e exatidão aceitas para as faixas de $\mu \mathrm{g} \mathrm{L}^{-1}$. A aplicação da metodologia a amostras reais permitiu detectar a presença de testosterona e estradiol tanto em amostra de esgoto doméstico bruto quanto tratado sinalizando a ineficiência do tratamento para estes analitos.

AGRADECIMENTOS: A PROPESP-IFSUL pelo apoio financeiro.

\section{REFERÊNCIAS}

ARAÚJO, J. C.. Estudo da eficiência do tratamento de efluentes domésticos da cidade de Araraquara/SP na remoção de hormônios sexuais. Dissertação (Mestrado em Química Analítica) - Instituto de Química de São Carlos, Universidade de São Paulo, São Carlos, 2006. DOI: http://doi.org/10.11606/D.75.2006.tde-17012007-144901

BARONTI, C.; CURINI, R.; D'ASCENZO, G.; DI CORCIA, A.; GENTILI, A.; SAMPERI, R.. Monitoring Natural and Synthetic Estrogens at Activated Sludge Sewage Treatment Plants and in a Receiving River Water. Environmental Science \& Technology, v.34, n.24, p.5059-5066, 2000. DOI: http://doi.org/10.1021/es001359q

BASHEER, C.; JAYARAMAN, A.; KEE, M. K.; VALIYAVEETTIL, S.; LEE, H. K.. Polymer-coated hollow-fiber microextraction of estrogens in water samples with analysis by gas chromatography-mass spectrometry. Journal of Chromatography A, v.1100, n.2, p.137-143, 2005. DOI: http://doi.org/10.1016/j.chroma.2005.09.039

BRITO, N. M.; AMARANTE JUNIOR, O. P.; POLESE, L.; RIBEIRO, M. L.. Validação de métodos analíticos: estratégia e discussão. Pesticidas: Revista de Ecotoxicologia e Meio Ambiente, v.13, 2003. DOI: http://dx.doi.org/10.5380/pes.v13i0.3173
CAMPOS, A. C. V.. Otimização e validação do método para análise de microcontaminantes de preocupação emergente por cromatografia gasosa acoplada à espectrometria de massas utilizando derivatização online. Dissertação (Mestrado em Química) - Universidade Federal de Ouro Preto, Ouro Preto, 2018.

CHRISTIAN, G. D.. Analytical Chemistry, Solutions Manual. 5 ed. Nova York: John Wiley \& Sons, 1994.

COSTA, A. H. L.. Análise energética in silico da interação do ER? Com estrogênios relacionados a neoplasma mamária: estradiol e dietilestilbestrol. Dissertação (Mestrado em Bioinformática) - Universidade Federal do Rio Grande do Norte, Natal, 2018.

DALLEGRAVE, A.. Determinação de hormônios estrógenos e progestágenos em amostras ambientais por GC/MS. Tese (Doutorado - Curso de Química) - Universidade Federal do Rio Grande do Sul Instituto de Química, Porto Alegre, 2013.

FERREIRA, A. P.. Desreguladores endócrinos em estações de tratamento de águas residuais de lodo: complicações ambientais. Acta Scientiarum Tecnologia, v.35, n.2, p.307316, 2012. DOI:

http://doi.org/10.4025/actascitechnol.v35i2.10619 
FILIPPE, T. C.. Cafeína, fármacos, hormônios e produtos de cuidados pessoais no Rio Palmital/PR. Dissertação (Mestrado em Ciências Ambientais) - Universidade Tecnológica Federal do Paraná, Curitiba, 2018.

GOEURY, K.; DUY, S. V.; MUNOZ, G.; PRÉVOST, M.; SAUVÉ, S. Data supporting the optimization of liquid chromatography tandem mass spectrometry conditions to analyze EPApriority hormones and bisphenol $A$ in water samples. Data in Brief, v.24, p.103958, 2019. DOI: http://doi.org/10.1016/ j.dib.2019.103958

HE, P.; AGA, D. S.. Comparison of GC-MS/MS and LC-MS/MS for the analysis of hormones and pesticides in surface waters: advantages and pitfalls. Analytical Methods, v.11, n.11, p.1436-1448, 2019. DOI:

http://doi.org/10.1039/C8AY02774A

INMETRO. Instituto Nacional de Metrologia, Qualidade e Tecnologia. Orientação sobre Validação de métodos analíticos. INMETRO, 2016.

IUPAC. International Union of Pure and Applied Chemistry. Compêndio de Terminologia Química. 2 ed. Oxford: Publicações científicas de Blackwell, 1997. DOI: http://doi.org/10.1351/goldbook

JAUKOVIĆ, Z. D.; GRUJIĆ, S. D.; BUJAGIĆ, I. V. M.; LAUŠEVIĆ, $M$. D.. Determination of sterols and steroid hormones in surface water and wastewater using liquid chromatographyatmospheric pressure chemical ionization-mass spectrometry. Microchemical Journal, v.135, p.39-47, 2017. DOI: http://doi.org/10.1016/j.microc.2017.07.011

JONHSON, A. C.; BELFROID, A.; DI CORCIA, A.. Estimating steroid oestrogen inputs into activated sludge treatment works and observations on their removal from the effluent. The Science of the Total Environment, v.256, n.2-3, p.163173, 2000. DOI: http://doi.org/10.1016/S00489697(00)00481-2

KOLPIN, D. W.; FURLONG, E. T.; MEYER, M. T.; THURMAN, E. M.; ZAUGG, S. D.; BARBER, L. B.; BUXTN, H. T.. Pharmaceuticals, hormones, and other organic wastewater contaminants in U.S. streams, 1999-2000: A National Reconnaissance. Environmental science \& technology, v.36, n.6, p.1202-1211, 2002. DOI:

http://doi.org/10.1021/es011055j

MORSELLI, E.; SANTOS, R.; CRIOLLO, A.; NELSON, D. M.; PALMER, F. B.; CLEGG, J. D.. The effects of oestrogens and their receptors on cardiometabolic health.2017. Nat. Rev. Endocrinol, v.13, p.352-364. DOI:

http://doi.org/10.1038/nrendo.2017.12
ORATA, F.. Derivatization Reactions and Reagents for Gas Chromatography Analysis. In: ADVANCED GAS CHROMATOGRAPHY - PROGRESS IN AGRICULTURAL, BIOMEDICAL AND INDUSTRIAL APPLICATIONS. Anais. 2012.

PESSOA, G. P.; SOUZA, N. C.; VIDAL, C. B.; ALVES, J. A. C.; FIRMINO, P. I. M.; NASCIMENTO, R. F.; SANTOS, A. B.. Occurrence and removal of estrogens in Brazilian wastewater treatment plants. The Science of the Total Environment, v.490, p.288-295, 2014. DOI: http://doi.org/10.1016/i.scitotenv.2014.05.008

QUEIROZ, M. E. C.; MELO, L. P.. Recentes avanços da in-tube SPME-LC para bioanálises. Scientia Chromatographica, v.5, n.3, p.167-179, 2013. DOI: http://doi.org/org/10.4322/sc.2014.002

SILVA, C. G. A.; COLLINS, C. H.. Aplicações de cromatografia líquida de alta eficiência para o estudo de poluentes orgânicos emergentes. Quím. Nova, São Paulo, v.34, n.4, p.665-676. DOI: http://doi.org/10.1590/S0100$\underline{40422011000400020}$

SOUZA, C. P. F. A.; FALQUETO, E.. Descarte de Medicamentos no Meio Ambiente no Brasil. Revista Brasileira de Farmácia, v.96, n.2, p.1142-1158, 2015.

TEIXEIRA, R. B.; MARQUES, C. A.; CARVALHO, N. R.; GOMES, L. E. T.; SILVA, F. T.; PAIVA, T. C. B.. Determinação de hormônios estrogênicos em esgoto bruto e efluente de uma estação descentralizada de tratamento por lodos ativados. Revista Ambiente e Água, v.13, n.2, 2018. DOI: http://doi.org/10.4136/ambi-agua.2059

TERNES, T. A.; STUMPF, M.; MUELLER, J.; HABERER, K.; WILKEN, R. D.; SERVOS, M.. Behavior and occurrence of estrogens in municipal sewage treatment plants I. Investigations in Germany, Canada and Brazil.1999. Science of The Total Environment, v.225, n.1-2, p.81-90. DOI: http://doi.org/10.1016/s0048-9697(98)00334-9

TUCK, S. P.; FRANCIS, R. M.. Testosterone, Bone and Osteoporosis. In: JONES, T. H.. Advances in the Management of Testosterone Deficiency, Frontiers of Hormone Research. Front Horm Res. Basel, Karger, v.37, p.123-132, 2008. DOI: http://doi.org/10.1159/000176049

VIALI, M. A.. Avaliação da eficiência de remoção de hormônios em estações de tratamento de efluentes. (Dissertação de graduação) - Universidade Federal de Juiz de Fora, Juiz de Fora, 2014.

Wood, R. How to validate analytical methods. TrAC Trends in Analytical Chemistry, v.18, n.9-10, p.624-632, 1999. DOI: http://doi.org/10.1016/s0165-9936(99)00150-8

A CBPC - Companhia Brasileira de Produção Científica (CNPJ: 11.221.422/0001-03) detém os direitos materiais desta publicação. Os direitos referem-se à publicação do trabalho em qualquer parte do mundo, incluindo os direitos às renovações, expansões e disseminações da contribuição, bem como outros direitos subsidiários. Todos os trabalhos publicados eletronicamente poderão posteriormente ser publicados em coletâneas impressas sob coordenação da Sustenere Publishing, da Companhia Brasileira de Produção Científica e seus parceiros autorizados. Os (as) autores (as) preservam os direitos autorais, mas não têm permissão para a publicação da contribuição em outro meio, impresso ou digital, em português ou em tradução. 